\title{
Conclusion des Journées Chevreul 2008
}

\author{
Anne LE GUILLOU \\ Fats and Oils senior expert, Danone Research \\ <anne.le-guillou@danone.com>
}

Je souhaite tout d'abord remercier l'ensemble des intervenants pour la qualité de leurs présentations, ainsi que M. Clavie de la société Perles de Gascogne, qui a fourni des huiles d'amandon de pruneau et de noisettes, nous permettant de déguster au restaurant Drouant un dîner de gala mémorable.

\section{Sources d'oméga-3 et d'oméga-6}

Le Pr P. Legrand (Agrocampus, INRA, Rennes) nous a fait un point des récentes avancées dans le domaine du métabolisme des oméga-3, en particulier du DHA, dont le rôle dans le bon fonctionnement du cerveau et du cœur n'est plus à démontrer. En effet, même en présence d'une quantité importante d'ALA, son taux de conversion est très faible chez l'Homme (évalué à moins de $1 \%$ ), d'où la nécessité d'apports directs de DHA par l'alimentation.

Comme chacun sait, la source principale de cet acide gras est la chair de certains poissons. Or, comme l'a bien montré S. Lozachmeur (Sté Polaris), les stocks de poissons ne sont pas en grande forme, et on estime qu'en 2010 la demande en huile de poissons sera supérieure à I'offre. Comment pallier ce manque? En assurant une meilleure gestion des stocks, en valorisant les coproduits (œufs et peaux de poissons), par une aquaculture bien maîtrisée...

Les huiles d'algues peuvent également permettre de compléter les besoins en DHA, comme nous I'a présenté le Dr M. Leblanc (Sté Lonza). Leur mode de production par biofermentation en fait une source végétale renouvelable.

Les huiles végétales ne contenant que les précurseurs de l'EPA et du DHA, et vu le faible taux de conversion, elles ne peuvent être qu'une réponse partielle au rééquilibrage de notre alimentation. J.M. Maurette (consultant) nous a notamment présenté les intérêts de l'huile de lin, riche en ALA, qui a reçu en 2006 un avis favorable de I'AFSSA concernant son alimentarité en version vierge (demande de la Sté Oenobiol - I'huile de lin raffinée a été autorisée en mélange en février 2008, suite à une demande de la filière).

Nous voyons également arriver de nouvelles huiles végétales, comme l'huile d'Echium présentée par le Dr M. Mir (Ste Croda Europe), en cours d'approbation Novel Foods, à la fois riche en oméga- 6 et oméga- 3 puisqu'elle qui combine de fortes teneurs en GLA et en SDA. Le SDA étant le précurseur immédiat de I'EPA, et cette étape métabolique ayant un meilleur rendement que le passage EPA à DHA, l'huile $d^{\prime} E c h i u m$ pourrait être intéressante à recommander pour des régimes végétariens.

M. Mallet (Sté Phythea, France) a illustré le rôle majeur des huiles marines et végétales utilisées dans les compléments alimentaires (ophtalmologie, santé osseuse, protection solaire, gestion du stress...). Ce marché a été multiplié par deux en 6 ans, représentant plus d'un milliard d'euros à la fin 2006. II devrait encore doubler d'ici à 2012 (étude Précepta).

\section{Lipides et dermatologie}

Avec l'exposé de C. Montastier (Sté Cometap, France), qui nous a fait partager sa passion pour la peau, nous avons pu voir l'importance des lipides, et en particulier des acides gras essentiels, dans le bon fonctionnement de notre peau. Les applications de crèmes riches en composés lipidiques sont tout à fait complémentaires d'apports spécifiques dans l'alimentation, comme également montré par le $\mathrm{Dr} \mathrm{T}$. Lassel (Sté Danone Research, France) avec les études cliniques réalisées avec le produit Essensis ${ }^{\circledR}$ de Danone, qui contient notamment de I'huile de bourrache riche en GLA (acide gamma linolénique). À noter le rôle clé de la matrice produit, puisque l'on obtient une meilleure biodisponibilité de l'huile émulsifiée dans un yaourt que seule.

Le GLA a été présenté par M. Van Hoorn (Sté Bioriginal Food \& Sciences, Pays-Bas) comme le « bon oméga- 6 ». En effet, si notre alimentation est globalement trop riche en oméga- 6 , elle peut être déficience en GLA du fait notamment d'un taux de conversion limité du LA. Des études montrent le rôle du GLA sur la santé de la peau, mais également sur l'état inflammatoire en général, en particulier les maladies coronaires et l'arthrite.

A. Castera (Iterg, France) a également présenté un tableau très complet des nouvelles huiles végétales disponibles pour des formulations cosmétiques, venant de nombreuses zones géographiques, avec des compositions en acides gras et en insaponifiables spécifiques très intéressantes.

Les huiles et la peau possèdent les mêmes composés naturels, de même que les lipides des plantes et de la peau jouent les mêmes rôles.

Cet exposé a été complété par celui de V. Rossow (Ets B. Rossow et $\mathrm{Cie}$ ), sur de nouveaux beurres et cires végétaux utilisés en cosmétique, répondant à des tendances " bio ", " exotiques ", notamment des « beurres » en provenance $d$ 'Inde (sal, kokum, mowrah). La tendance est de substituer des ingrédients peu appréciés des consommateurs (lanoline).

\section{Huiles à bénéfices mixtes}

Le $\operatorname{Pr}$ Z. Charouf (Faculté des Sciences, Université Mohammed V, Rabat) nous a montré l'importance du développement durable lié à la production de l'huile d'argane, avec la mise en place d'un réseau de coopérative de femmes au Maroc, mettant en avant les enjeux socioéconomiques du développement de l'arganeraie marocaine. Cette huile a de nombreux intérêts, à la fois en cosmétique et en alimentation.

Avec également des applications cosmétiques et alimentaires, une huile de germe de blé obtenue par pression à froid Viogerm ${ }^{\circledR}$ nous a été présentée par M. Genet (Sté Hochdorf Nutrition), qui a également insisté sur la richesse de cette huile en composés à haute valeur nutritionnelle comme la vitamine $\mathrm{E}$, les phytosterols et les phospholipides.

Les $\operatorname{Dr}$ A. Saunois et D. Daguet (Laboratoires Expansciene) ont eux aussi insisté sur la richesse de l'insaponifiable de leurs huiles de colza et de maïs concentrées obtenues par 
distillation moléculaire, une technologie « propre ». Avec une quantité moindre de matière grasse, il est possible d'enrichir un aliment en composés sous forme native ayant un rôle physiologique clé.

À partir d'huiles plus connues, comme le colza ou le tournesol, il est possible par sélection variétale traditionnelle d'obtenir des variétés plus adaptées à certaines applications. P. Lesigne (Sté Monsanto International) nous a notamment présenté des huiles riches en acide oléique plus résistantes à l'oxydation, à teneur réduite en saturés et permettant de réduire la formation d'AGT.

\section{Lipides et satiété}

Le Pr P. Besnard (Ensbana, UMR Inserm U866) nous a tout d'abord présenté l'influence non seulement de l'odeur et de la texture des lipides, mais aussi de leur goût «biochimique», sur la régulation de la prise alimentaire. Des résultats récents suggèrent que la gustation est impliquée dans la consommation excessive de lipides, en plus des perceptions tactiles et olfactives associées au plaisir.

Puis nous avons eu 2 présentations sur des nouveaux ingrédients :

Le Dr H. Keizer (Lipid Nutrition, IOI Group, Pays-Bas) nous a parlé de PinnoThin ${ }^{\mathrm{TM}}$, huile de pin de Corée riche en acide pinolenique, acide gras de la famille des oméga-6, qui a montré lors d'études cliniques son effet sur les hormones liées à la satiété (augmentation de la production de GLP-1 et de CCK) ainsi qu'une réduction de la prise alimentaire et une baisse de poids corporel associée.

Enfin, le Dr P. Rijken (DSM Food Specialties, Pays-Bas) a présenté Fabuless ${ }^{\top \mathrm{M}}$, un ingrédient constitué d'une émulsion de fraction d'huiles de palme et d'avoine. La qualité de l'émulsion lui permet de ne pas être totalement détruite par les lipases, et l'arrivée de lipides non digérés dans l'iléon génère des signaux de satiété. Les produits laitiers sont un véhicule intéressant et approprié pour cet ingrédient.

\section{Conclusion}

Concernant les applications alimentaires, comme nous l'a rappelé $C$. Kohler (Conseil Alimentation Santé), il convient avant tout lancement d'un nouvel ingrédient de s'assurer de son statut réglementaire, afin de vérifier si un dossier Novel Food est ou non nécessaire. Importance de la législation, qui peut être vue comme un frein à l'innovation, mais qui garantit la sécurité du consommateur.

Nous avons pu voir que les huiles et leurs dérivés sont largement concernés par cette procédure, avec notamment durant ces 2 jours les exemples de l'huile de lin, de l'huile $d^{\prime}$ Echium, des concentrés d'huile de colza et de germe de maïs. Certaines huiles échappent au statut de Novel Food, du fait d'une utilisation traditionnelle locale, comme c'est le cas de I'huile de pin de Corée.

Si notre diète est trop riche en lipides, les preuves sont de plus en plus nombreuses pour démontrer que la quantité n'est pas le seul facteur à prendre en compte, mais que la qualité des lipides est également fondamentale. Ainsi, de même que tous les oméga-3 ne sont pas équivalents (ALA, EPA, DHA), tous les oméga- 6 ne sont pas équivalents (GLA, pinolénique). Tous les acides gras saturés non plus, comme le rappelait $\mathrm{P}$. Legrand avec le rôle de I'acide myristique dans le métabolisme des $\mathrm{O} 3$. Dans la lutte contre l'obésité, nous avons également vu le rôle des lipides sur leur propre métabolisme, et leur influence sur la régulation hormonale de l'appétit et de la prise de poids. Bien qu'étant de nature lipidique, de nouveaux ingrédients semblent avoir une action positive sur la gestion du poids.

Nous avons vu aussi que les compléments alimentaires et les aliments peuvent jouer des rôles complémentaires pour le rééquilibrage de la diète.
De même, traitements cosmétiques et alimentation peuvent permettre une approche globale et synergique des problématiques liées à la peau et son équilibre. C'est ce qu'A. Castera a nommé dermo- et nutri-cosmétique, inside and outside nutrition.

Les aliments fonctionnels vont donc vraisemblablement continuer à se développer avec succès, en particulier dans le domaine de la dermonutrition.

Les fournisseurs découvrent ou redécouvrent de nombreuses sources de lipides fonctionnels, on peut citer: fenugrec, baobab, amarante, pépins de grenade, bourrache, buruti, ciste, kokum, mowrah, sal, echium..., qui permettent des bénéfices multiples et des approches marketing innovantes.

Le rôle des constituants dits "mineurs", tels que caroténoïdes, polyphenols, squalène..., a été également mis en avant à de nombreuses reprises.

Dans tous les domaines d'application, et comme l'on souligné plusieurs intervenants, il faut noter l'importance de la préservation et de la meilleure exploitation des ressources halieutiques, mais aussi des sources végétales terrestres.

Ces aspects de développement durable et de biodiversité sont maintenant pris en compte quasi systématiquement, que ce soit en alimentaire, pour les compléments ou en cosmétique. Ceci est particulièrement vrai dans le cas de sources provenant de cultures à faibles échelles (ex : argan, baobab...), dans des pays émergents où les aspects socioéconomiques sont clés.

Ces journées ont également été l'occasion de rendre un chaleureux hommage à la mémoire de Michel Ollivon, disparu I'an dernier (In memoriam dans $\mathrm{OCL}, 2007, \mathrm{n}^{\circ} 5$, p. 257), en lui remettant la médaille Chevreul à titre posthume en présence de son épouse, de ses collègues et amis. 